\title{
MR Study of Myocardial Fiber Structure Using Diffusion Tensor Imaging
}

\author{
Yin $\mathrm{Wu}^{1,2,3,4}$ and $\mathrm{Ed} \mathrm{X} . \mathrm{Wu}^{1,2}$ \\ ${ }^{1}$ Dept. of Electrical and Electronic Engineering, ${ }^{2}$ Laboratory of Biomedical Imaging and Signal \\ Processing, The University of Hong Kong, Hong Kong \\ ${ }^{3}$ Paul C. Lauterbur Research Center for Biomedical Imaging, Institute of Biomedical and Health \\ Engineering, Shenzhen Institute of Advanced Technology, ${ }^{4}$ Key Laboratory of Biomedical Informatics \\ and Health Engineering, Chinese Academy of Sciences, Shenzhen, China
}

\begin{abstract}
This paper discussed the left ventricular myocardium structure in both normal and pathological states using diffusion tensor imaging. Myocardial fiber pathway distribution was investigated in normal canine hearts, and effects of infarct size and location on myocardium structure remodeling were studied in $L A D$ - and $L C X$-related myocardium infarct (MI) porcine models. Majority fiber pathways were found to have small helix angles and dominate the fiber architecture in normal myocardium. While in the two MI groups studied, substantial fiber quality degraded in infarct region with significant correlation with infarct size. The double-helical structure shifted towards more left-handed, especially in LAD-related MI group. The results suggest that both infarct size and location affect the myocardium structural remodeling. These studies not only provide supplemental information on myocardial fiber architecture and cardiac mechanics in normal hearts, but also revealed the alterations in myocardial fiber quality and structure in infarct hearts.
\end{abstract}

\section{Introduction}

It has been proved that left ventricular (LV) myocardium structure plays a critical role in determining both mechanical contraction [1-3] and electrical activation [4]. Therefore, a precise description of myocardial fiber structure of LV wall can lead to better understanding of cardiac mechanics and electrical conduction in both normal and pathologic states. Most research on myocardial fiber structure focuses on its orientation, which is one of the critical determinants of myocardial wall motions and represents the direction where the current spreading is the most rapid [5,6]. Myocardial wall was first studied by means of histological measurements and a well-ordered distribution was observed $[7,8]$. In recent years, diffusion tensor imaging (DTI) has emerged as a powerful tool for rapid measurement of cardiac geometry and fiber structure at high spatial resolution. Numerous DTI studies show that the orientation of myocardial fibers changes smoothly from a left-handed helix in the epicardium to a right-handed helix in the endocardium, and the transmural helix angle typically range from $-60^{\circ}$ at the epicardium to $+60^{\circ}$ at the endocardium when viewed from base [9-11]. However, a complete knowledge of the contractile behavior of cardiac muscle also includes individual cardiac muscle length which is a key factor in characterizing the contractility of single cardiac muscle [12]. Globally, the distribution of myocardial fiber distribution and pathway can play a determinant role in the overall LV contraction. Therefore, investigation on myocardial fiber pathway may provide supplementary information on fiber architecture and cardiac mechanics [13, 14].

However, LV remodeling not only involves functional but also structural alterations in infarct myocardium [15]. Such changes have been found to occur in infarct, adjacent and remote myocardium [16-18]. Several ex vivo DTI studies have been performed to investigate myocardium microstructure degradation on infarct hearts samples. Increasing of apparent diffusion coefficient (ADC) and reduction of fractional anisotropy (FA) in infarct region were usually obtained $[19,20]$. More recently, in vivo DTI was successfully applied to investigate fiber architecture remodeling in a group of patients with acute myocardium infarct (MI) in various LV locations [21]. The results suggested an alteration of double-helical fiber structure besides changes of FA and ADC. But the influence of MI location, one of possible determinants of LV remodeling [22], on myocardium structure was not discussed. So far, the influence of infarct location on cardiac remodeling is still controversial. Some researchers reported that infarct, not the location, is the dominant determinant of LV remodeling [23, 24]. However, others believed that anterior MI was associated with greater LV remodeling compared with nonanterior infarction $[25,26]$. In this paper two groups of porcine model with septal or lateral transmural MI induced by LAD or LCX ligation were examined for functional and fiber structural alterations in $\operatorname{LV}[27,28]$. The effects of 
infarct locations and size on myocardium structure and fiber architecture alterations were discussed from DTI studies.

\section{Methods}

All MR experiments were conducted on a Philips 3T scanner.

\subsection{Myocardial fiber pathway length distribution}

Normal canine hearts $(N=6,30-35 \mathrm{~kg})$ were excised, arrested in diastole, and fixed with formalin. LV short-axis (SA) planes were prescribed as perpendicular to long axis and covered the entire heart. DTI was performed using single-shot spin echo EPI (SE-EPI) sequence: $\mathrm{TE}=45 \mathrm{~ms}$, $\mathrm{TR}=4 \mathrm{~s}$, diffusion sensitivity of $\mathrm{b}=800 \mathrm{~s} / \mathrm{mm}^{2}$, gradient direction $=15$, slice number $\approx 45$, spatial resolution of 1.13 $\mathrm{mm}^{3}$ with zero slice gap, NEX $=40$, total scan time was $\sim 1 \mathrm{hr}$ per sample. 3D reconstruction of LV myocardial fiber pathways were obtained by using PRIDE software package. A MATLAB program was developed to compute the fiber orientation and pathway length. Myocardial fiber helix angle was computed as defined by Scollan DF et al [10] (Fig.1). The distribution of number of fibers tracked within each slice and their average pathway length was calculated respectively as a function of the helix angle using $9^{\circ}$ steps.

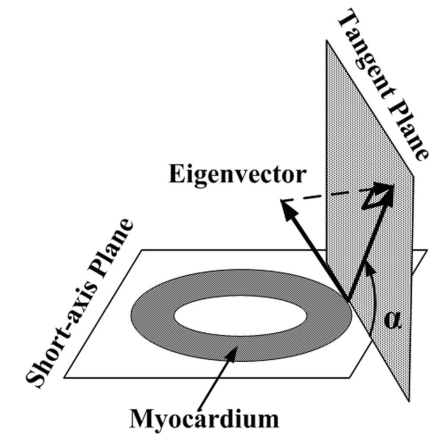

Fig.1 Helix angle definition.

\subsection{Myocardium structure remodeling in MI model}

2.2.1. Surgery procedure. Adult mini-pigs $(\mathrm{N}=6$ for each infarct group) were anesthetized via IV administration of propofol. LAD or LCX coronary artery distal to the first diagonal branch was occluded with balloon to create infarct group \#1 or infarct group \#2. Six normal mini-pigs of similar weight and age were used as controls.

2.2.2. In vivo CMR imaging. Thirteen weeks after surgeries, all infarct animals together with 2 controls were performed CMR imaging. Eight SA slices covering the whole heart were imaged using and ECG-triggered breathheld balanced-FFE cine sequence with parameters: TR/TE= $5.5 / 2.2 \mathrm{~ms}$, slice thickness $=8 \mathrm{~mm}$ with 0 slice gap, cardiac frames $=20$, in-plane resolution $=1.04 \times 1.04 \mathrm{~mm}^{2}$, flip angle $=45^{\circ}$, and total scan time $\approx 1 \mathrm{~min}$. Delay-enhanced T1weighted images were obtained on the same slices about 10 min after a bolus IV injection of Gd using an IR-M2D-FFE sequence with parameters: $\mathrm{TR} / \mathrm{TE}=3.8 / 1.3 \mathrm{~ms}$, IR time $=$ $275 \mathrm{~ms}$, in-plane resolution $=0.68 \times 0.68 \mathrm{~mm}^{2}$, flip angle $=$ $15^{\circ}$, and total acquisition time $\approx 5$ min. Ejection fraction $(\mathrm{EF})$ values and infarct size were analyzed using Segment and correlated among the animals scanned.

2.2.3. Ex vivo DTI study. All animals were sacrificed after in vivo study. The fixation procedure of heart samples and the DTI study were the same as those described in 2.1. 3D myocardial fiber tracks were visualized using PRIDE. DTI parameters, i.e. FA, mean $\mathrm{ADC}$, eigenvalues and eigenvectors, were measured using DtiStudio. Ten slices covering infarction were selected and segmented into infarct, adjacent and remote regions [28, 29]. Fiber quality described by FA, mean ADC, axial and radial diffusivities were measured in all regions. Histogram of helix angle distribution was analyzed using $10^{\circ}$ steps. Three groups of fibers were classified: left-handed helical fiber (LHF) with helix angle within $-90^{\circ}$ to $-30^{\circ}$, circumferential fiber (CF) within $-30^{\circ}$ to $30^{\circ}$, and right-handed helical fiber (RHF) within $30^{\circ}$ to $90^{\circ}$ [21]. Percentages of three groups of fibers were computed in adjacent and remote regions.

2.2.4. Histological analysis. After ex vivo DTI, portions of the slices with infarction were selected, from which approximate $1 \mathrm{~cm}^{2}$ pieces adjacent and remote to the infarct were cut out and embedded in paraffin. Then, they were sectioned into $5 \mu \mathrm{m}$ slices and stained with Masson's trichrome for myocytes and connective tissue to visualize the myocardial structure integrity.

\section{Results}

\subsection{Myocardial fiber pathway length distribution}

Fiber distribution was investigated with FA and direction thresholds set at 0.15 and $40^{\circ}$, respectively. Average fiber pathway length distribution in eight representative slices among the six normal heart samples as a function of the fiber helix angle was examined (Fig.2a). Fiber pathways at middle or upper ventricle are much longer than those near apex. In each slice, the long fiber pathways usually have small helix angles, and fiber pathways with larger helix angles are usually shorter. By weighting the fiber pathway length by respective fiber number (Fig.2b), total fiber pathway length was obtained (Fig.2c). For each SA slice, the percentages of the total fiber pathway length occupied by those tracked fibers with helix angle within $\pm 20^{\circ}$ were decreased from base to apex. These results indicated that fibers with small helix angle dominate the myocardial fiber architecture in terms of total length of all fiber pathways crossing each SA slice, and this dominance near apex is not as great as that observed in myocardium closer to the base. 
Similar distribution pattern was observed although fiber pathway length was affected by different FA and directional threshold.

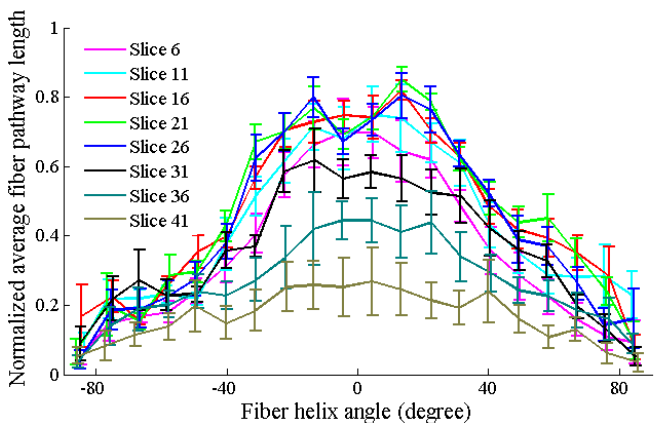

a

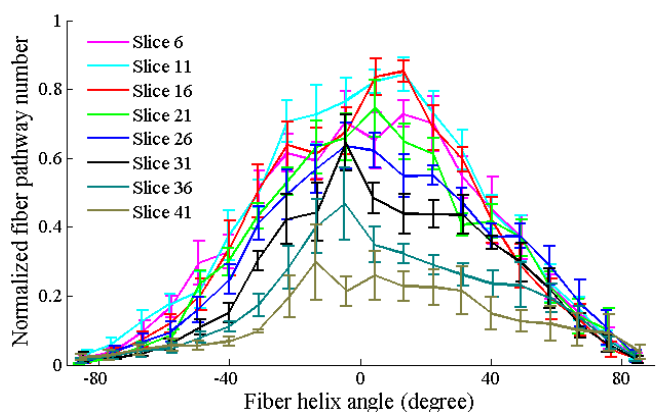

$\mathbf{b}_{1}$

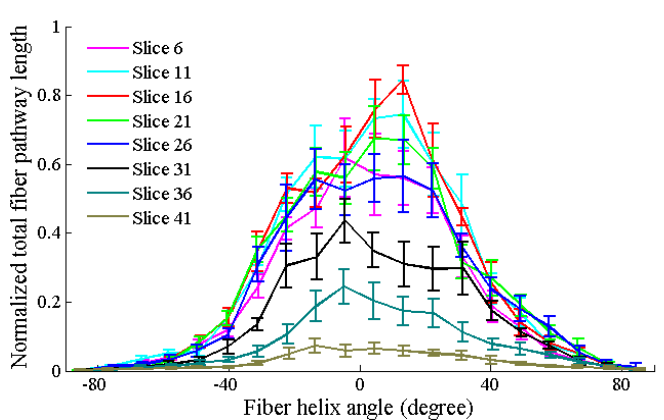

c

Fig.2 Average myocardial fiber pathway length (a), respective fiber pathway number (b), and total fiber pathway length (c) distribution as function of fiber helix angle.

\subsection{Cardiac functional remodeling}

LV EF was found to decrease with increase of infarct size in both MI groups, yielding $\mathrm{R}=-0.90,-0.91$ for $\mathrm{LAD}-$ and LCX-related MI groups, respectively (Fig.3).

\subsection{Myocardium structural remodeling}

3.3.1 Myocardial fiber quality degradation. Both infarctions clearly disrupt the continuity of the fibers near infarct region. Significant decrease of FA was observed in infarct region, but not in adjacent and remote regions compared with controls. Between the two MI groups, no substantial difference was obtained in all regions (Fig.4). Mean ADC increased significantly only in infarct region and no substantial changes were observed in all regions between two MI groups. Similar results were obtained for axial and radial diffusivities. FA values correlated significantly with infarct size in infarct region, yielding similar negative coefficients in both MI groups (Fig.5). And positive correlations were exhibited for mean ADC, axial and radial diffusivities with infarct size in both MI groups.

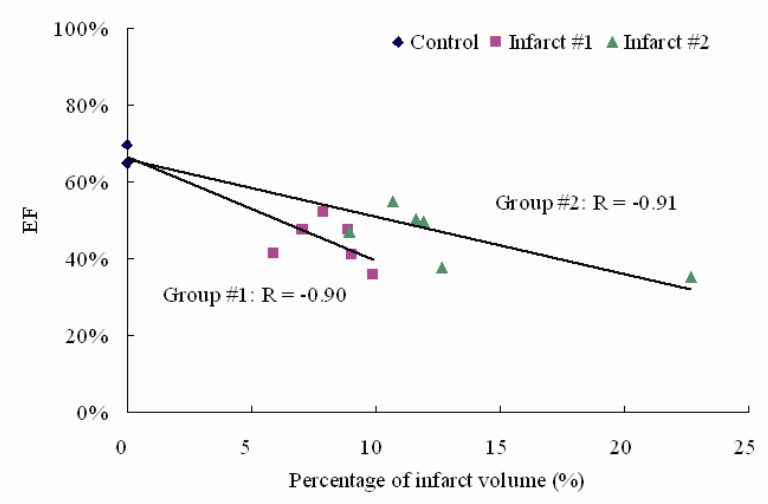

Fig.3 LV EF as a function of infarct volume percentages.

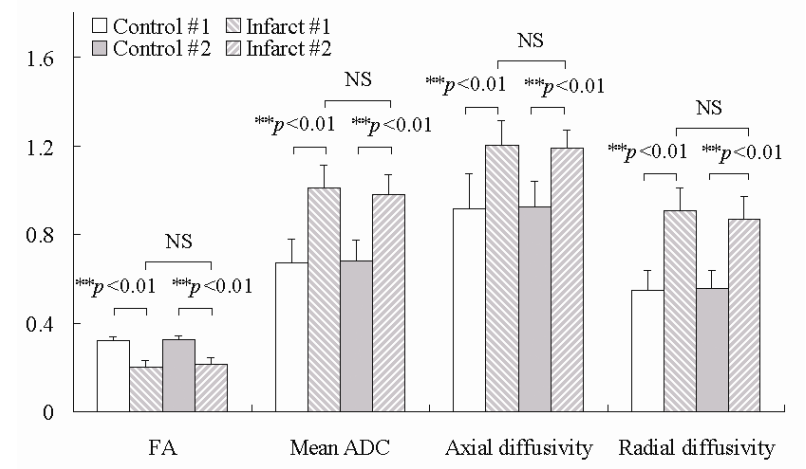

Fig.4 FA, mean ADC, axial diffusivity and radial diffusivity in infarct region of two infarct groups and their controls. The unit of mean ADC, axial and radial diffusivities is $\times 10^{-3} \mathrm{~mm}^{2} / \mathrm{s}$.

3.3.2 Myocardial fiber orientation alteration. In adjacent region, helix angle distribution was found to shift towards left-handed in both MI groups. LHF and CF of LAD-related MI group were significantly different from control and LCX-related MI group (Fig.6a). But no substantial changes of 3 groups of fibers were found in LCX-related MI group. However, in remote region, no significant fiber distribution change was observed in both MI groups (Fig.6b).

3.3.3. Histological analysis. The typical Masson's trichrome-stained views of the myocardial tissue in regions adjacent to the infarct were illustrated (Fig.7). The adjacent regions exhibited substantial tearing of the myocardial fiber bundles with extended formation of fibrosis. But in the remote region, such tearing and fibrosis could not be observed clearly. 


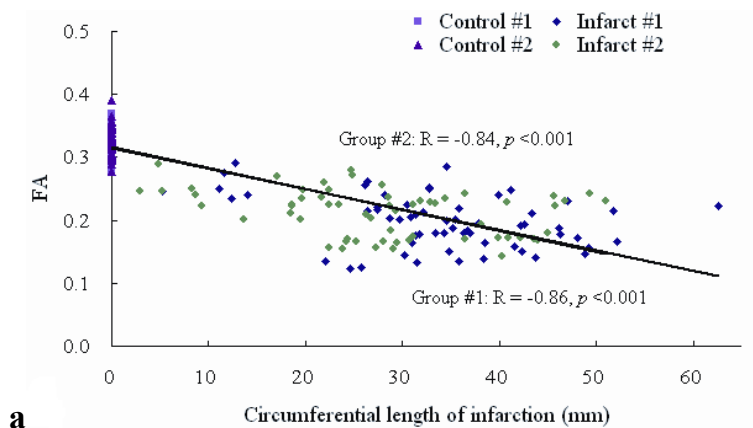

$\mathbf{a}$

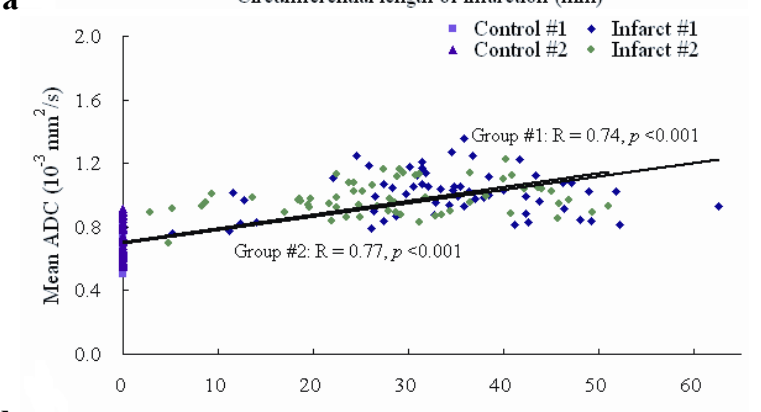

b
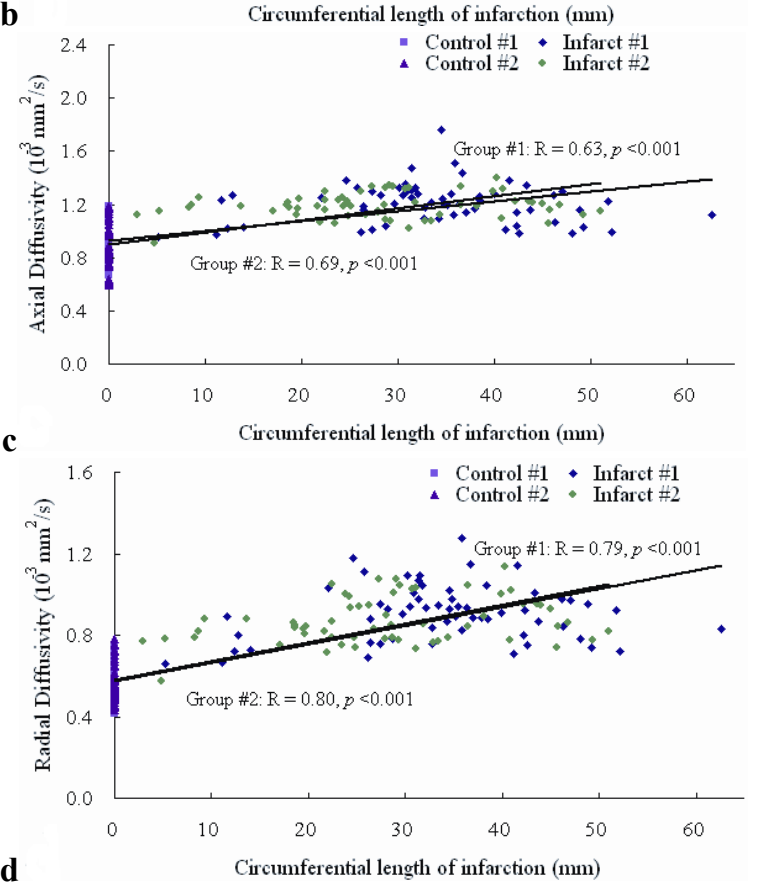

Fig.5 Correlation of FA (a), mean ADC (b), axial (c) and radial (d) diffusivity with epicardial circumferential length of infarction.

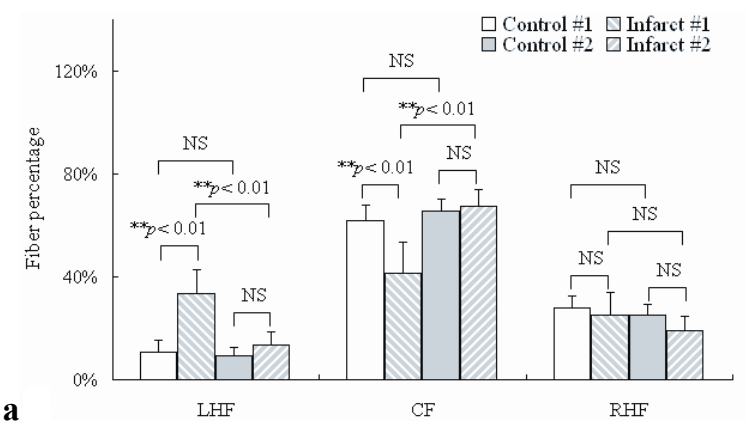

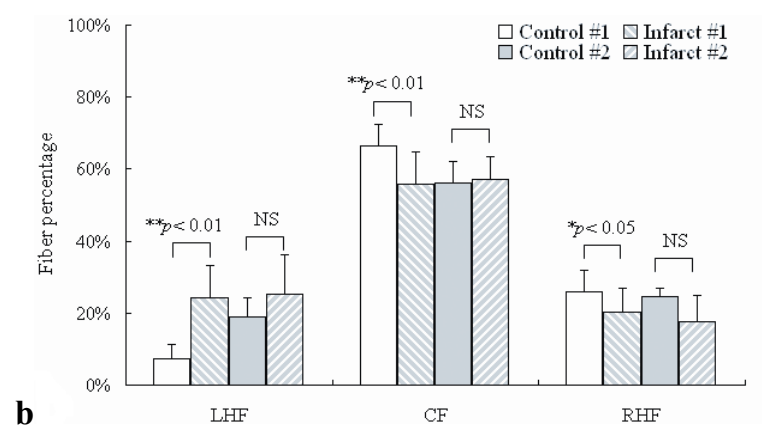

Fig.6 Percentages of LHF, CF and RHF in adjacent (a) and remote (b) regions of two infarct and control groups.

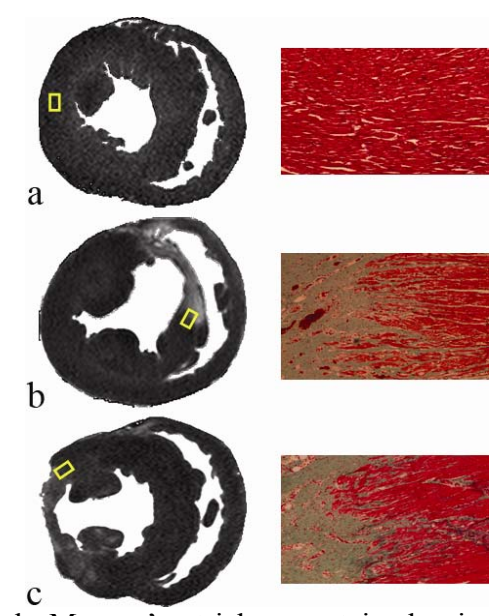

Fig.7 Typical Masson's trichrome-stained views of middle myocardium tissues (yellow box) selected from control (a), LADrelated MI (Infarct \#1) (b) and LCX-related MI (Infarct \#2) (c).

\section{Discussions and Conclusions}

In normal hearts, most fiber bundles, particularly those having long pathways in myocardium, tend to have small helix angles and spiral circumferentially in mid-wall. The fiber pathways at middle and upper ventricle are longer than those near apex. The findings suggest that these mid-wall fibers dominate the fiber architecture in myocardium. Such characteristics may contribute significantly to the highly efficient and effective myocardial contraction and explain the predominant deformation in LV is radially-oriented during cardiac contraction. Such myocardial fiber pathway and connectivity characterization using DTI may provide insights into myocardial fiber architecture and cardiac mechanics in both normal and diseased hearts.

In MI hearts, myocardium structure remodeling was observed concurrent with well-known cardiac function deterioration. EF decreased with increase of infarct size. Changes of DTI parameters, such as FA, mean ADC, axial and radial diffusivities were revealed compared with respective controls, especially substantial degradation in infarct region. But no significant difference was observed between two MI groups in terms of fiber quality. All DTI parameters correlated well with infarct size in infarct region. 
These results suggested that degradation of myocardium structure is infarct size dependent. But for myocardial fiber architecture, the double-helical fiber distribution was found to shift towards more left-handed compared with controls, especially in LAD-related MI group. This fiber architecture alteration is associated with infarct location. In general, MI heart structure remodeling involves myocardial fiber quality degradation and fiber architecture alteration. And this myocardium remodeling is determined by both infarct size and location.

\section{Acknowledgments}

This work was supported by a research grant from Hong Kong Research Grant Council (GRF HKU7794/07M).

\section{References}

[1] T. Arts, et al, "A model of the mechanics of the left ventricle," Ann Biomed Eng, vol. 7, pp. 299-318, 1979.

[2] F. E. Rademakers, W. J. Rogers, et al, "Relation of regional cross-fiber shortening to wall thickening in the intact heart. Three-dimensional strain analysis by NMR tagging," Circulation, vol. 89, pp. 1174-82, Mar 1994.

[3] L. A. Taber, M. Yang, et al, "Mechanics of ventricular torsion," J Biomech, vol. 29, pp. 745-52, Jun 1996.

[4] O. A. Smiseth and E. W. Remme, "Regional left ventricular electric and mechanical activation and relaxation," J Am Coll Cardiol, vol. 47, pp. 173-4, Jan 32006.

[5] P. C. Franzone, L. Guerri, M. Pennacchio, and B. Taccardi, "Spread of excitation in 3-D models of the anisotropic cardiac tissue. II. Effects of fiber architecture and ventricular geometry," Math Biosci, vol. 147, pp. 131-71, Jan 151998.

[6] A. Kanai, et al "Optical mapping reveals that repolarization spreads anisotropically and is guided by fiber orientation in guinea pig hearts," Circ Res, vol. 77, pp. 784-802, Oct 1995.

[7] D. D. Streeter, Jr., H. M. Spotnitz, et al, "Fiber orientation in the canine left ventricle during diastole and systole," Circ Res, vol. 24, pp. 339-47, Mar 1969.

[8] D. D. Streeter, Jr. and W. T. Hanna, "Engineering mechanics for successive states in canine left ventricular myocardium. II. Fiber angle and sarcomere length," Circ Res, vol. 33, pp. 65664, Dec 1973.

[9] E. W. Hsu, et al, "Magnetic resonance myocardial fiberorientation mapping with direct histological correlation," Am J Physiol, vol. 274, pp. H1627-34, May 1998.

[10] D. F. Scollan, A. Holmes, R. Winslow, and J. Forder, "Histological validation of myocardial microstructure obtained from diffusion tensor magnetic resonance imaging," Am J Physiol, vol. 275, pp. H2308-18, Dec 1998.

[11] Y. Wu and E. X. Wu, "MR study of postnatal development of myocardial structure and left ventricular function," Journal of Magnetic Resonance Imaging, vol. (in press), 2009.

[12] A. Manring and P. A. Anderson, "The contractility of cardiac muscle," Crit Rev Bioeng, vol. 4, pp. 165-201, 1980.

[13] Y. Wu, E. X. Wu, et al, "Myocardial Fiber Length Mapping with MR Diffusion Tensor Imaging," Conf Proc IEEE Eng Med Biol Soc, vol. 7, pp. 7004-7, 2005.
[14] E. X. Wu, Y. Wu, et al, "Study of myocardial fiber pathway using magnetic resonance diffusion tensor imaging," Magn Reson Imaging, vol. 25, pp. 1048-57, Sep 2007.

[15] P. P. Sengupta, J. Korinek, et al, "Left ventricular structure and function: basic science for cardiac imaging," $\mathrm{J} \mathrm{Am} \mathrm{Coll}$ Cardiol, vol. 48, pp. 1988-2001, Nov 212006.

[16] H. F. Weisman, D. E. Bush, et al, "Global cardiac remodeling after acute myocardial infarction: a study in the rat model," $J$ Am Coll Cardiol, vol. 5, pp. 1355-62, Jun 1985.

[17] J. Zhang, N. Wilke, et al, "Functional and bioenergetic consequences of postinfarction left ventricular remodeling in a new porcine model. MRI and 31 P-MRS study," Circulation, vol. 94, pp. 1089-100, Sep 11996.

[18] J. Bogaert, H. Bosmans, et al, "Remote myocardial dysfunction after acute anterior myocardial infarction: impact of left ventricular shape on regional function: a magnetic resonance myocardial tagging study," J Am Coll Cardiol, vol. 35, pp. 1525-34, May 2000.

[19] E. W. Hsu, R. Xue, A. Holmes, and J. R. Forder, "Delayed reduction of tissue water diffusion after myocardial ischemia," Am J Physiol, vol. 275, pp. H697-702, Aug 1998.

[20] J. Chen, S. K. Song, et al, "Remodeling of cardiac fiber structure after infarction in rats quantified with diffusion tensor MRI," Am J Physiol Heart Circ Physiol, vol. 285, pp. H946-54, Sep 2003.

[21] M. T. Wu, W. Y. Tseng, et al, "Diffusion tensor magnetic resonance imaging mapping the fiber architecture remodeling in human myocardium after infarction: correlation with viability and wall motion," Circulation, vol. 114, pp. 1036-45, Sep 52006.

[22] R. G. McKay, M. A. Pfeffer, et al, "Left ventricular remodeling after myocardial infarction: a corollary to infarct expansion," Circulation, vol. 74, pp. 693-702, Oct 1986.

[23] R. Fox, A. H. Hakki, A. S. Iskandrian, and S. Kane, "Location of myocardial necrosis as an independent determinant of left ventricular performance: analysis of 96 patients," $\mathrm{Am} \mathrm{J}$ Cardiol, vol. 53, pp. 483-6, Feb 11984.

[24]S. Orn, C. Manhenke, et al, "Effect of left ventricular scar size, location, and transmurality on left ventricular remodeling with healed myocardial infarction," Am J Cardiol, vol. 99, pp. 1109-14, Apr 152007.

[25] M. J. Gotte, A. C. van Rossum, et al, "Recognition of infarct localization by specific changes in intramural myocardial mechanics," Am Heart J, vol. 138, pp. 1038-45, Dec 1999.

[26] S. Thanavaro, R. E. Kleiger, et al, "Effect of infarct location on the in-hospital prognosis of patients with first transmural myocardial infarction," Circulation, vol. 66, pp. 742-7, Oct 1982.

[27] Y. Wu, H. F. Tse, and E. X. Wu, "Diffusion tensor MRI study of myocardium structural remodeling after infarction in porcine model," Conf Proc IEEE Eng Med Biol Soc, vol. 1, pp. 1069-72, 2006.

[28] E. X. Wu, Y. Wu, et al, "MR diffusion tensor imaging study of postinfarct myocardium structural remodeling in a porcine model," Magn Reson Med, vol. 58, pp. 687-95, Oct 2007.

[29] Y. Wu, C. W. Chan, et al, "MR study of the effect of infarct size and location on left ventricular functional and microstructural alterations in porcine models," J Magn Reson Imaging, vol. 29, pp. 305-12, Feb 2009. 